\title{
Computer-aided procedure for optimization of layer thickness uniformity in thermal evaporation physical vapor deposition chambers for lens coating
}

\author{
Salvador Bosch \\ Departament de Física Aplicada i Electrònica, Universitat de Barcelona, Diagonal 647, \\ 08028 Barcelona, Spain
}

(Received 27 March 1991; accepted 14September 1991)

\begin{abstract}
A computer-aided method to improve the thickness uniformity attainable when coating multiple substrates inside a thermal evaporation physical vapor deposition unit is presented. The study is developed for the classical spherical (dome-shaped) calotte and also for a plane sector reversible holder setup. This second arrangement is very useful for coating both sides of the substrate, such as antireflection multilayers on lenses. The design of static correcting shutters for both kinds of configurations is also discussed. Some results of using the method are presented as an illustration.
\end{abstract}

\section{INTRODUCTION}

Technical production of thin film coatings for lenses requires the use of big high vacuum evaporation plants in order to simultaneously coat a large number of lenses. Nevertheless, it is necessary to obtain sufficient uniformity in the deposition of materials over all the substrates being coated. For this purpose, in physical vapor deposition (PVD) using crucibles or electron beam guns, lenses are held on suitable palettes which are rotated with respect to a vertical axis passing through the center of the chamber while the evaporation is carried out.

Normally, spherical segments are used because they give better thickness uniformity than plane palettes. To increase the final uniformity, static correction shutters of appropriate position, shape and size must be designed. Other major factors to obtain good thickness uniformity are (a) to locate the evaporation source at the best radial distance from the center of the chamber; (b) to carefully monitor the evaporation rate, keeping it as uniform as possible in order to maintain the shape of the vapor cloud emitted from the crucible.

In many practical applications it is necessary to coat both sides of the substrates, e.g., broadband antireflection treatments on lenses. Thus, a further improvement for industrial purposes is to coat the two surfaces without opening the vacuum chamber. This greatly reduces the total process time and there is therefore increasing interest in reversible substrate holders. These usually consist of several ( 7 or 8 ) plane sectors assembled (at a certain angle) over a central axis (see Fig. 3). Using this equipment, the coating of one side is made while the whole setup is rotating around the axis of the chamber. Then, the evaporation process is stopped, the plane sectors are turned upside down and the other substrate side is similarly coated.

Although this setup may be built in such a way that it is closely adapted to the shape of a spherical segment spatial distribution, the lack of rotational symmetry makes a perfect thickness uniformity mathematically impossible, even using correcting static shutters during evaporation. From a practical point of view, the limiting thickness uniformity when coating a large number of substrates using standard industrial high vacuum equipment is about $\pm 1 \% .^{1}$ We will take this thickness uniformity as our practical limit. Moreover, it may be considered optimal when dealing with antireflective (AR) coatings involving several layers in the $100 \mathrm{~nm}$ range.

In this work, after a particular study of the geometrical arrangement corresponding to this reversible plane sector setup, we develop a method to obtain optimum layer thickness uniformity between the different coated substrates. The method will be useful both for the spherical and plane-sectors calotte. The geometrical study will take into account the influence of the shape of the vapor cloud emanating from the source and will allow the design of static correcting shutters for final thickness optimization.

\section{GEOMETRICAL RELATIONS}

Let us deduce the basic equation for all the computation methods that will follow. We shall assume that the evaporation source may be considered as a single surface element $d S$ (see Fig. 1) emitting towards the upper-half space in such a way that its emissive power depends only on the angle $\alpha$ with respect to its perpendicular direction. We shall take this dependence as directly proportional to the value $\cos ^{n}(\alpha), n$ being a value that depends on the evaporation conditions (material, type of crucible, applied evaporation power, etc.). ${ }^{1}$ Moreover, the source will be horizontal, i.e., the angle $\alpha$ will always be measured with respect to the vertical direction.

The supposition concerning the size of the source could be refined, but for a big PVD unit where the distance from the source to the substrates is almost $1 \mathrm{~m}$, it is enough to consider the crucible or electron beam gun as a single surface element.

The emissive characteristics of the source may also be slightly refined by introducing the isotropic component of the distribution, $A$, which changes the angular dependence to $(1+A) \cos ^{n}(\alpha)+A,{ }^{1}$ but the practical results obtained are similar to our simpler model.

A surface element to be coated $d A$ (Fig. 1), at a distance $d$ from the source and with its normal forming an angle $\beta$ with the line $d S-d A$, will subtend (from $d S$ ) a solid angle $d w=d A \cos (\beta) / d^{2}$. Then, the amount of evaporated material deposited onto the surface element $d A$ per unit time is 


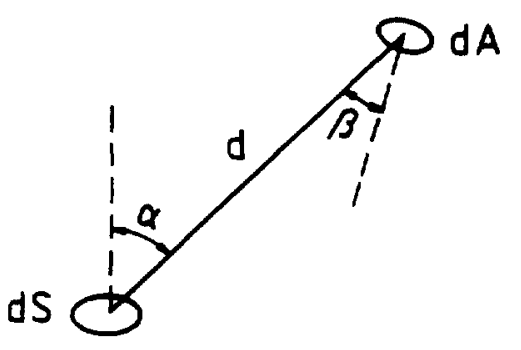

Fig. 1. Elementary geometrical configuration.

$$
d m=K \cos ^{n}(\alpha) d A \cos (\beta) / d^{2},
$$

where $K$ is a constant value that may also include the sticking coefficient between substrate and material.

All practical PVD configurations can be treated as a process of many differential depositions $d m$ with the same value $K$, provided the evaporation rate is maintained. The remaining problem is to adequately describe the simultaneous variations of $d, \alpha, \beta, \ldots$ as the evaporation process is taking place. Two main configurations are interesting: spherical segment substrate holder (dome-shaped calotte) and plane sector reversible holder, both rotating with respect to a vertical central axis during evaporation. The first is known to be far superior to plane holders. ${ }^{1,2}$ The second setup allows the coating of the two sides without opening the chamber.

Of course, we need only to compute the thickness deposited at a single holder turn, because all depositions take place with the holder rotating and differences between successive turns are only expected when evaporation rate monitoring fails.

Finally, it is important to notice that our supposition concerning the a priori knowledge of the exponent $n$ is not realistic. From a practical point of view, the operating method will be to compare the expected thickness values for different exponents $n$ with the experimental measurements of samples coated with a well known geometrical arrangement (using the numerical methods explained below). This comparison will enable us to deduce the emissive characteristics of the source under our evaporation conditions. Moreover, the value $n$ is taken to be constant during layer deposition, neglecting variations due to the reduction in the amount of evaporation material, for example. This practical procedure is mainly justified because of the assumed constant evaporation rate (well monitored) and also by the use of new and equally well filled crucibles. The remaining variations in $n$ may be considered a secondary effect, as we are dealing with $\pm 1 \%$ thickness accuracy within layers usually thinner than $1000 \AA$.

\section{A. Dome-shaped (spherical) calotte}

This evaporator geometry has been studied elsewhere. ${ }^{1}$ The basic configuration is shown in Fig. 2. The setup is fully defined by the height $h$, the radius of the dome $(\overline{O R}=\overline{P R})$ and the position of the evaporation source $Q$. With respect to expression (1), the angles $\alpha$ and $\beta$ are shown and $d=\overline{Q P}$. Using the mathematical expressions given in Ref. 1 it is possible to evaluate the thickness deposited at any

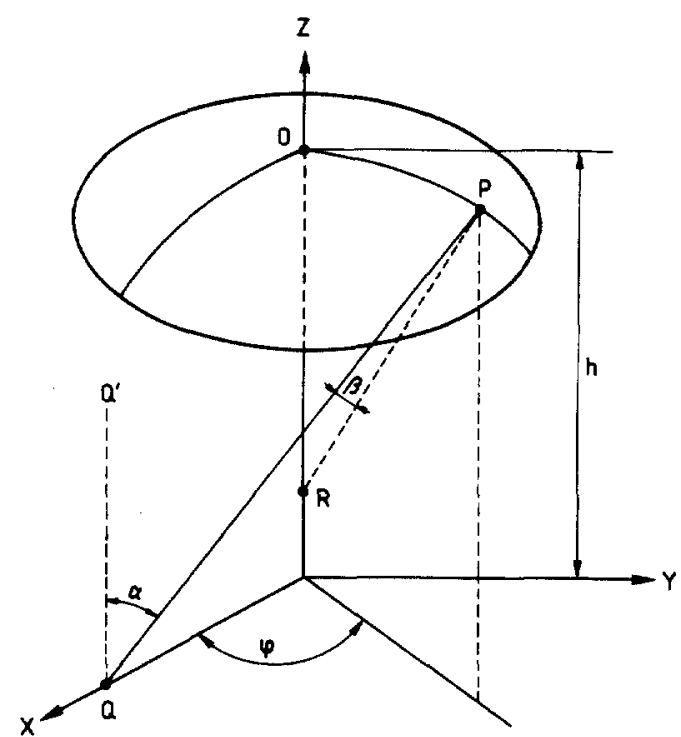

FIG. 2. Diagram of the dome-shaped calotte.

radial position $P$ of the substrate holder during any variation in angle $\varphi$. This may be used to optimize the position of the evaporation source and to design the static correcting shutters for optimum thickness uniformity. All the substrates placed on the calotte at the same radial distance from the rotation axis $(Z)$ are equivalent. This implies that, from the mathematical point of view, the perfect thickness uniformity would be attained using a well designed static correcting shutter during evaporation.

\section{B. Plane sectors reversible holder}

The sketch of this setup is presented in Fig. 3(a).

Let us designate by $\delta$ the angle between the rotation axis and the plane of an individual sector. Notice that, after the coating of one face and before the second one, the sectors will be turned upside down around the axis $O N$. For this configuration, the position of the substrate cannot be defined only by its distance from the rotation axis. A method to compute the thickness received by each point of one sector of the holder is developed in the following. Let us define [see Fig. 3(a)] the position $P$ of the receiving element $d A$ by the distances $\overline{P N}$ and $\overline{N O}$, all measured over the plane of the sector. Taking the evaporation source $d S$ placed at a point $Q$ on the $X$ axis, when the rotation of the sector is defined by $\varphi$, we have for the terms in expression (1)

$$
\alpha=\widehat{Q^{\prime} Q P}, \quad \beta=\widehat{Q P R}, \quad d=\overline{Q P},
$$

$Q^{\prime}$ being just any point above $Q$ and $R$ any point on the normal to the sector through $P$.

A further refinement can be incorporated in the computer program: to include the case of an imperfect sector reversion (which will be useful for comparison with experimental results). We may admit that a line lying on the plane of the sector and perpendicular to the axis $O N$ may not be horizontal [Fig. 3(b)] but tilted by a small angle $\theta$. For this more general case the true receiving point $P$ corresponds to a nontilted case point $P^{\prime}$. Once the distances $\overline{N O}, \overline{P N} \equiv \overline{P^{\prime} N}$ 


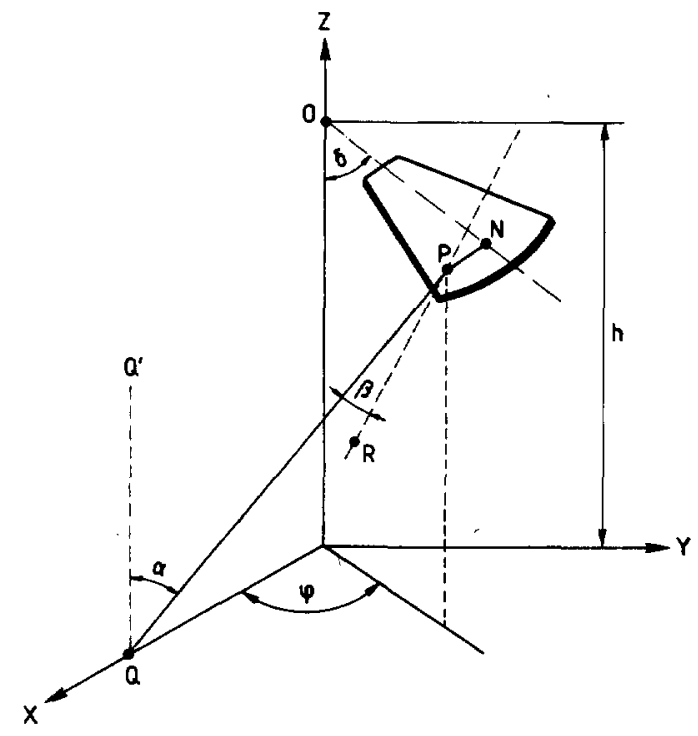

(a)

(b)

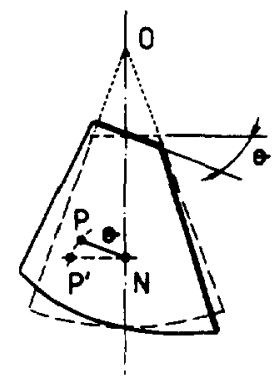

FIG. 3. Plane-sectors reversible lens holder setup.

and the angles $\theta$ and $\varphi$ are known, we may calculate the coordinates of $P$ from the following conditions:

$$
\begin{aligned}
& \mathbf{P N} \cdot \mathbf{O N}=0, \\
& \mathbf{P N} \cdot \mathbf{P}^{\prime} \mathbf{N}=\overline{\mathbf{P N}} \cdot \overline{\mathbf{P}^{\prime} \mathbf{N}} \cdot \cos (\theta), \\
& |\mathbf{P N}|=\overline{\mathbf{P N}}=\overline{\mathbf{P}^{\prime} \mathbf{N}} .
\end{aligned}
$$

Then we can find a point $R$ (which defines the angle $\beta$ ) in the normal line to the sector through the point $P$ by means of the following equations:

$$
\begin{aligned}
& R=P+\mathbf{P N} \times \mathbf{O N}, \text { for } \theta \text { as in Fig. } 3(\mathrm{~b}) ; \\
& R=P+\mathbf{N P} \times \mathbf{O N} \text {, for opposite tilting. }
\end{aligned}
$$

Finally, to compute relative thicknesses, it is only necessary to sum for the full interval $\varphi^{\prime}<\varphi<\varphi^{\prime \prime}$ where deposition takes place $\left(\varphi^{\prime}=0\right.$ and $\varphi^{\prime \prime}=2 \pi$ for rotating holder without static shutter). It is interesting to note that using this kind of reversal substrate holder, the two half cycles $0<\varphi<\pi$ and $\pi<\varphi<2 \pi$ give different deposited thicknesses, even when $\theta=0$. This is easily proved by computing both half cycles or noting the nonequivalent progressions of angle $\beta$. Of course this is not the case for the spherical sector.

Following the mathematical approach given above, one may readily develop computer programs to estimate the relative thickness deposited in any point of either the spherical or the plane-sectors calotte, corresponding to any change in the rotation of the holder (defined by the limiting angles $\varphi^{\prime}$ and $\varphi^{\prime \prime}$ ).

It is apparent in this development that we do not take into account the curvature of the surfaces of the coated lenses, assuming plane substrates lying tangentially to the holder. It would be easy to show that this curvature greatly influences the thicknesses deposited, since it greatly changes the angle $\beta$. Nevertheless, as the thickness over a particular substrate (lens) may not be optimized with our general methods (intended for the whole calotte) and the curvatures have a wide range of variation (from concave to convex), it would be only of mathematical interest to deal with nonplane substrates.

\section{OPTIMIZATION PROCEDURE}

The optimization method proposed comprises the following three consecutive steps:

(i) determination of the emissive characteristics of the source,

(ii) determination of the best source position,

(iii) design of static correcting shutters.

Of course, a precise measurement of all mechanical elements of the evaporation unit which define our geometrical configuration is initially assumed. Namely, the radius of the spherical calotte and the height of the rotation point $O$, for the dome-shaped holder. For the reversible system: the angle $\delta$, the height of $O$ and the distances $N$ and $P$ over the plane sector which define the points of interest. Then, the first step in our procedure is to characterize the source (exponent $n$ ) under the working conditions: vacuum pressure, evaporation rate, crucible shape and size, etc. This may be accomplished by performing a test deposition with the evaporation source placed at any well defined radial position and comparing the experimental thicknesses obtained at different points on the calotte (either spherical or reversible) with the expected values for that source position under different emanating conditions (values of $n$ ). In practice, one would select several points on which to place the test substrates. Remember that the holder always rotates during evaporations. The exponent that best fits the measured values is selected to represent the source.

Once the directional emissive characteristics of the source are known, the next factor to decide is the best radial position

TABLE I. Points of calculation for the reversible holder.

\begin{tabular}{lrrrrrrrrrr}
\hline \hline Point designation & 0 & 1 & 2 & 3 & 4 & $4^{\prime}$ & 5 & 6 & 7 & $7^{\prime}$ \\
\hline Distance $(O-N)(\mathrm{cm})$ & 17.0 & 20.0 & 23.0 & 29.0 & 31.5 & 31.5 & 36.0 & 39.0 & 40.0 & 40.0 \\
Distance $(P-N)(\mathrm{cm})$ & 0.0 & 3.5 & -4.0 & 0.0 & -7.5 & 7.5 & -4.0 & 3.0 & -10.6 & 10.6 \\
\hline
\end{tabular}




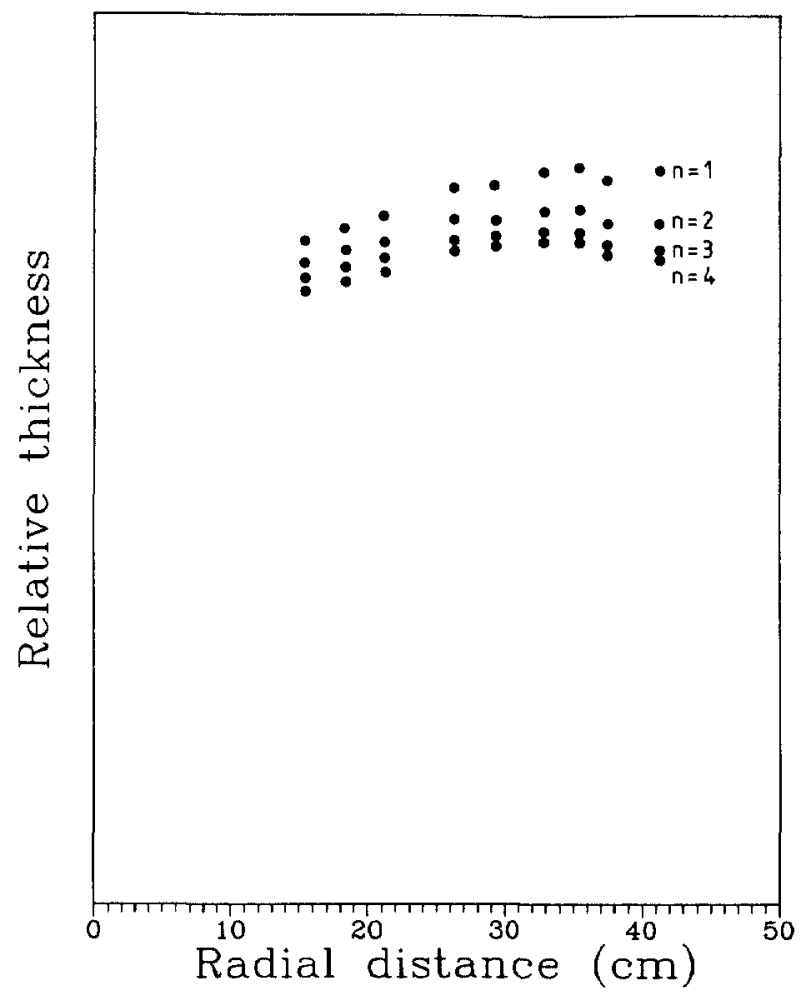

FIG. 4. Calculated relative thicknesses in the points $0,1, \ldots$ and 8 of Table I when $n=1,2,3,4$; the evaporation source is at $36 \mathrm{~cm}$ from the rotation axis.

for the source. Naturally, this is the point that gives the best thickness uniformity for our geometrical arrangement without using any correcting shutter. In practice, this is the source position which gives the same thickness at the innermost and outermost usable points of the calotte. This choice is easy with the help of the simulation programs devised following the guidelines explained above. It is only necessary to find the thickness distributions over several points of the substrate holder for some radial positions of the evaporation source, every $1 \mathrm{~cm}$ for example (note that $n$ is already known). When comparing these results the selection will be obvious. In fact, this step of the optimization method may be skipped, because its effect may be compensated by the correcting shutter. However, it is always more convenient to work with the evaporation source giving the best uniformity and this is easy with the help of the computation programs.
The design of static correcting shutters is based on the assumption that atoms and molecules emitted by the source travel in straight lines to the substrates. This is a logical supposition for the typical values of pressure and source-substrate distances in high vacuum chambers. ${ }^{3}$

If a coating includes several layers of different materials, it may be a good operating method to design independent correcting shutters for each source. This allows independent compensations in case of very different materials. Then, a standard independent correcting shutter must be placed above the source, so that other sources are not influenced.

Working with $n$ fixed (i.e., fixed evaporation conditions) the form of the appropriate static correcting shutter for a given calotte is only dependent on the radial position of the crucible. As it is designed to equate all the thicknesses, a logical method to conceive the shutter is to take the minimum thickness obtained without shutter (of course, for the same crucible position) as the target value. The design procedure will slightly differ for the two types of holders (always assuming continuous rotation of the holder during evaporations). For the spherical calotte, the starting thickness distribution (without any shutter) is calculated (for the chosen source position and estimated value $n$ ). The minimum thickness value obtained should not be reduced, so the shutter must not screen the corresponding point of the sector. Moreover, it has to shadow the other points until the obtained thickness is always the same (i.e., this initial minimum). As the shutter is above the source, we have to compute the elementary thicknesses obtained for angles $\varphi$ beginning from $\pi$ and going to 0 (or $2 \pi$, see Fig. 2) until their sum reaches the chosen minimum value. This $\varphi$ value defines the limit of half the shutter. The exact shape and size will depend on the plane selected on which to place it, i.e., will be determined by the intersection between this plane and the line from the source to the limiting point of the holder to be screened. As already explained, because of the rotational symmetry, it is mathematically possible to obtain a perfectly uniform thickness distribution using this kind of static correcting shutter. Thus, the final limiting uniformity using a well designed shutter is our practical limiting value $( \pm 1 \%)$.

For reversible rotating calottes, a design procedure consistent with the established premises may be as follows. First, use the corresponding computer program to evaluate the thickness distribution over the different substrates of a single sector, choosing only the positions of their centers. The min-

TABLE II. Relative thicknesses (with respect to position 3) in the points of the reversible holder for different source properties (value $n$ ).

\begin{tabular}{|c|c|c|c|c|c|c|c|c|c|c|c|}
\hline Point designation & 0 & 1 & 2 & 3 & 4 & $4^{\prime}$ & 5 & 6 & 7 & $7^{\prime}$ & 8 \\
\hline$\%$ thickness $(n=1)$ & -7.2 & -5.4 & -3.5 & 0.0 & 0.4 & 0.4 & 2.5 & 3.2 & 1.5 & 1.5 & 2.9 \\
\hline$(n=2)$ & -5.8 & -4.3 & -2.8 & 0.0 & 0.2 & 0.2 & 1.6 & 1.8 & -0.2 & -0.2 & -0.1 \\
\hline$(n=3)$ & -5.4 & -4.0 & -2.7 & 0.0 & 0.3 & 0.3 & 1.5 & 1.5 & -0.6 & -0.6 & -1.3 \\
\hline$(n=4)$ & -5.9 & -4.4 & -3.0 & 0.0 & 0.7 & 0.7 & 2.0 & 2.0 & -0.1 & -0.1 & -1.4 \\
\hline
\end{tabular}


TABLE III. Thickness and refractive index obtained at the points of the reversible holder. They are ellipsometric measurements at $546.1 \mathrm{~nm}$ from a test evaporation of a high index material. Points 0 and 8 did not have any substrate.

\begin{tabular}{lccccccccc}
\hline \hline Point & 1 & 2 & 3 & 4 & 4 & 5 & 6 & 7 & $7^{\prime}$ \\
\hline Thickness $(\AA)$ & 775 & 788 & 808 & 811 & 814 & 822 & 820 & 803 \\
Ref. index & 2.114 & 2.127 & 2.137 & 2.143 & 2.142 & 2.157 & 2.159 & 2.143 & 2.158 \\
\% thickness & -4.1 & -2.5 & 0.0 & 0.4 & 0.7 & 1.8 & 1.5 & -0.6 & -0.3 \\
\hline
\end{tabular}

imum of all these values will be the thickness deposited for all the points considered if we shadow the sector during evaporation with a similar procedure as above. Recalling Fig. 3, the computation method has to be consistent with the continuous rotation of the holder during evaporation while taking into account the nonequivalent turns $0 \leqslant \varphi \leqslant \pi$ and $\pi \leqslant \varphi \leqslant 2 \pi$. Thus, the sum of elementary thicknesses begins at $\varphi=\pi$ going to 0 and $2 \pi$ in regular alternative steps $\Delta \varphi$ : $\pi+\Delta \varphi, \quad \pi-\Delta \varphi, \quad \pi+2 \Delta \varphi, \quad \pi-2 \Delta \varphi, \quad \pi+3 \Delta \varphi, \ldots$, ( $\Delta \varphi=0.5^{\circ}$ for example). This will compensate for the two different semi-cycles. When the sum attains the chosen minimum value, the two $\varphi$ angles $\left(\varphi_{1}\right.$ near 0 and $\varphi_{2}$ near $\left.2 \pi\right)$ define the limits of the static correcting shutter. Note that with this computation method $\left|\left(\varphi_{1}-0\right)-\left(2 \pi-\varphi_{2}\right)\right|$

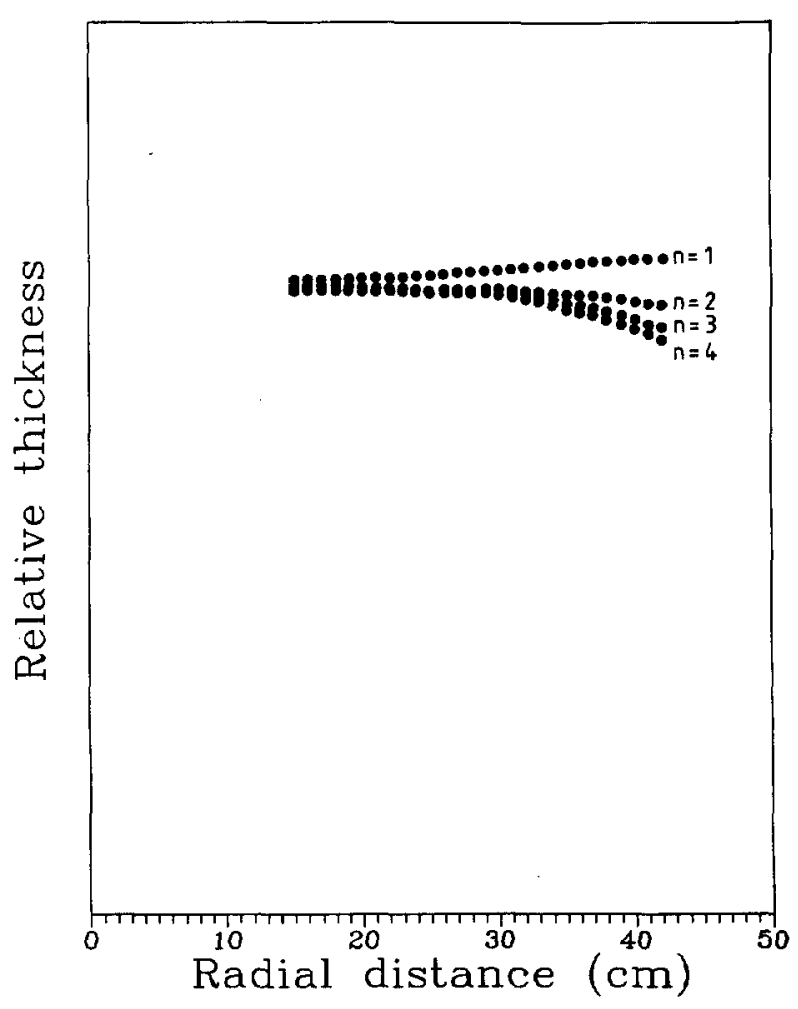

Fig. 5. Calculated relative thicknesses for our spherical sector when $n=1$, $2,3,4$; the evaporation source is $36 \mathrm{~cm}$ from the rotation axis and radial distances on the calotte range from 15 to $42 \mathrm{~cm}$ in $1 \mathrm{~cm}$ steps.
$=\Delta \varphi$, giving a correcting shutter which is to be manufactured symmetric about the radial line (see Fig. 7 ) provided $\Delta \varphi$ is small. The shape and size is found as before: will be determined by the intersection between the plane of the shutter and the line from the source to the limiting points of the holder to be screened. The number of points per sector is not limited by the method, but, as sectors continuously rotate around the vertical axis during deposition, it has to be pointed out that in the design of a correcting static shutter for final uniformity improvement it will only be possible to modify one thickness for each radial distance to the rotation axis. Our practical design procedure has been to match the thicknesses at the center of each substrate.

\section{AN EXAMPLE}

The method has been routinely used for process optimization in lens coating plants. The practical results are satisfactory. There are some important experimental details to be checked: avoid screening from the evaporation source to the calotte due to any mechanical element inside the vacuum chamber, avoid reflection of evaporated species on the walls of the chamber, etc. The practical agreement between theoretical computations and measured values of intermediate and final thickness distributions must always be judged within the $\pm 1 \%$ limit, as commented in the introduction. Nevertheless, it is worth noting that the optimizing method is indeed a valuable tool for industrial purposes, since it saves a lot of time and effort when adjusting new process conditions in a vacuum chamber. Initial thickness uniformity is usually poor (differences greater than $10 \%$ ) and, with the proposed method, it may be optimized with a minimum number of test evaporations.

In the subsequent figures we show some representative results. We shall illustrate the method by comparing the reversible calotte with the dome-shaped holder. Table I shows the distances $\overline{P N}$ and $\overline{N O}$ which define the positions of the center of nine lenses placed over a single sector of the reversing holder. The innermost (called 0 ) and outermost (called 8 ) limiting points of the sectors are also included. There are two pairs of symmetrical lenses about the $O N$ axis: $4-4^{\prime}$ and $7-7^{\prime}$. The angle $\delta$ was $65^{\circ}$ and the height $h$ of the point 0 above the source was $70 \mathrm{~cm}$.

Figure 4 compares the expected thickness distributions for several source characteristics $n=1,2,3,4$. The distance from the source to the rotation axis is taken to be $36 \mathrm{~cm}$. The graph is a plot of the relative thicknesses (only values for the 
TABLE IV. Relative thicknesses (with respect to position 3) in the points of the reversible holder for $n=3$ when $\theta=3$ deg.

\begin{tabular}{|c|c|c|c|c|c|c|c|c|c|c|c|}
\hline Point designation & 0 & 1 & 2 & 3 & 4 & $4^{\prime}$ & 5 & 6 & 7 & $7^{\prime}$ & 8 \\
\hline \% thickness & -5.4 & -3.8 & -3.0 & 0.0 & -0.5 & 1.1 & 1.0 & 1.9 & -2.1 & 0.8 & -1.3 \\
\hline
\end{tabular}

TABLE V. Relative thicknesses (with respect to position 3 ) in the points of the reversible holder for different source-axis distances (always for $n=3$ and $\theta=0^{\circ}$ ).

\begin{tabular}{|c|c|c|c|c|c|c|c|c|c|c|c|}
\hline Point designation & 0 & 1 & 2 & 3 & 4 & $4^{\prime}$ & 5 & 6 & 7 & $7^{\prime}$ & 8 \\
\hline$\%$ thickness $(33 \mathrm{~cm})$ & -3.9 & -2.8 & -2.5 & 0.0 & -2.0 & -2.0 & -0.9 & -1.8 & -5.8 & -5.8 & -7.4 \\
\hline$\%$ thickness $(34 \mathrm{~cm})$ & -4.4 & -3.2 & -2.9 & 0.0 & -1.7 & -1.7 & -0.2 & -0.7 & -4.5 & -4.5 & -5.4 \\
\hline$\%$ thickness $(35 \mathrm{~cm})$ & -4.9 & -3.6 & -3.2 & 0.0 & -1.4 & -1.4 & 0.5 & 0.4 & -3.1 & -3.1 & -3.4 \\
\hline
\end{tabular}

same $n$ can be compared) versus the radial distance from the points $0, \ldots, 8$ to the $Z$ axis. Differences with respect to position 3 are given as a percentage in Table II. Measuring experimental thicknesses, the value $n$ may be found by any best fitting procedure. Table III shows the thicknesses corresponding to a practical test for a high refractive index material deposited at $\approx 6 \AA / s$. We will assume $n=3$ for all the following computations. Further very careful experiments would be necessary to refine this value because of the close thickness results which $n=2$ or $n=4$ would give (see Table II). But this also shows that $n=3$ will give sufficient precision for the desired uniformity.

For comparison, Fig. 5 shows the corresponding distributions for a spherical calotte with radius $65.5 \mathrm{~cm}$ and highest point 0 placed $65.5 \mathrm{~cm}$ above the source, which would be a dome approaching the form and size of the previous reversible holder. The source is also $36 \mathrm{~cm}$ from the axis. Radial distances on the calotte range from 15 to $42 \mathrm{~cm}$ in $1 \mathrm{~cm}$ steps. By contrast with the previous holder, observation of Fig. 5 reveals that the optimum evaporation source position would have to be further than $36 \mathrm{~cm}$ from the rotation axis (for $n=3$ ) in order to increase the thickness at high radial distances most naturally.

In Table IV we see the same type of computed results as in Table II but for the reversible lens holder when it was tilted $3^{\circ}$ during evaporation (with $n=3$ ). Note the differences between positions 4-4' and 7-7'. This kind of data can become useful in assessing the cosine-exponent $(n)$ for the evaporation source. If a different thickness is obtained in practice in positions 4 and $4^{\prime}$, and 7 and $7^{\prime}$, postulating a tilting angle one may draw meaningful conclusions from an otherwise useless experiment (if all the values agree within the assumption, of course). We may also conclude that the tilting of the sector during the evaporation corresponding to Table III was negligible.

Table V shows the expected distributions ( with $n=3$ ) for different source-axis distances. Therefore, the optimum source position for this reversible holder is $34.5 \mathrm{~cm}$. Similar calculations for the spherical calotte yield an optimum evaporation source position at $40 \mathrm{~cm}$ from the rotation axis. Figure 6 corresponds to this situation, where all the thicknesses differ in less than $1 \%$ (compare relative heights). So, no correcting shutter is needed for the spherical calotte if the source is placed in this position.

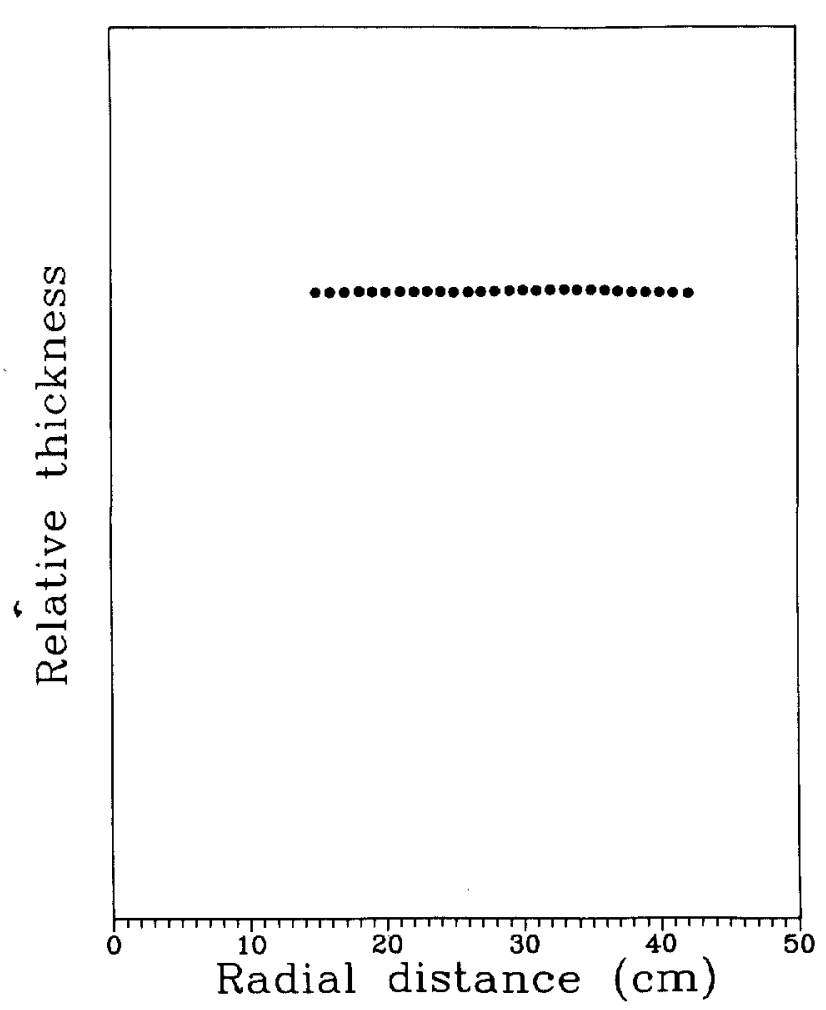

FIG. 6. Calculated relative thicknesses for our spherical sector $(n=3)$ when the evaporation source is at $40 \mathrm{~cm}$ from the rotation axis. 


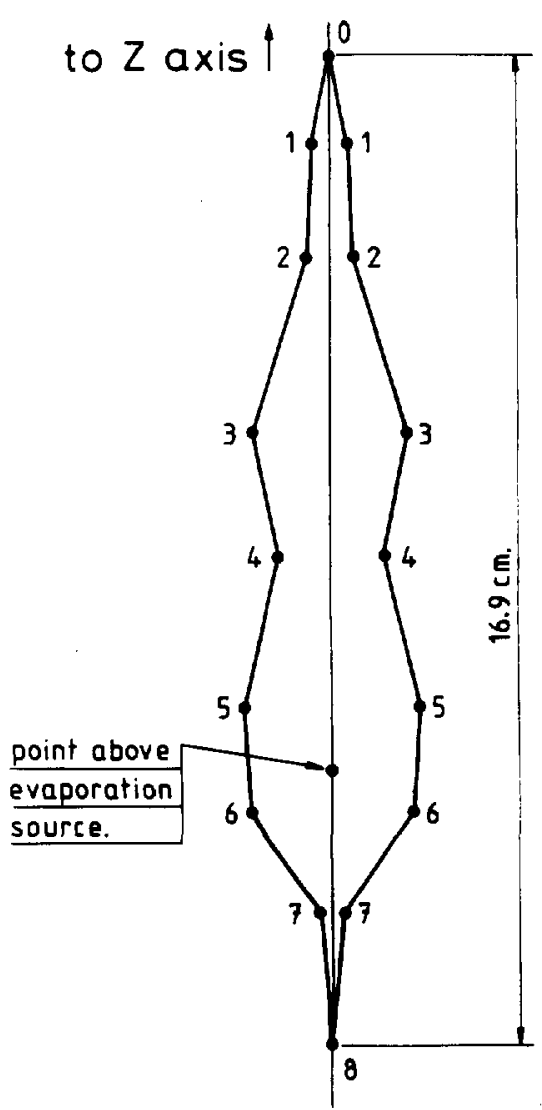

FIG. 7. Designed correcting shutter for the reversible holder. The evaporation source position is $34.5 \mathrm{~cm}$ away from the vertical rotation axis and the shutter is horizontally placed $40 \mathrm{~cm}$ above the source. The vertices are designed to give equal thicknesses at the points of Table I.
In Fig. 7 we see the designed correcting shutter for the reversible holder with the source in the computed optimum position (always for $n=3$ and $\theta=0$ ). It was calculated to be horizontally placed $40 \mathrm{~cm}$ above the source, pointing to the rotation axis. The mark on the central line of the shutter indicates the point exactly above the source and may be helpful to position it. Using this shutter, the final thickness at the center of each lens is optimized. Other points are not very different because the substrates are small.

\section{CONCLUSIONS}

Computation methods to simulate the thickness obtained over the substrate holder of a thermal evaporation PVD unit have been developed. These include the two main types of holders for lens coating: spherical sector and plane-sectors reversible calotte. This enables us to devise a method to optimize the thickness distribution obtained for the different points of the substrate holder. It comprises the following steps: characterize the emissive properties of the source, find the best radial position for it and design a static correcting shutter for a final improvement in thickness uniformity.

${ }^{1}$ H. K. Pulker, in Coatings on Glass (Elsevier, Amsterdam, 1984), Chap. 6, pp. 169-191.

${ }^{2}$ A. H. Macleod, in Thin Films Optical Filters (Adam Hilger, Bristol, 1987), Chap. 4.

${ }^{3}$ See, for example, R. W. Berry, P. M. Hall, and M. T. Harris, Thin Film Technology (Krieger, Huntington, NY, 1979), Chap. 2. 